Revista Médica Sinergia

Vol. 6, Núm. 8, agosto 2021, e705

\title{
Manejo farmacológico del dolor crónico en el embarazo
}

Pharmacological management of chronic pain in pregnancy

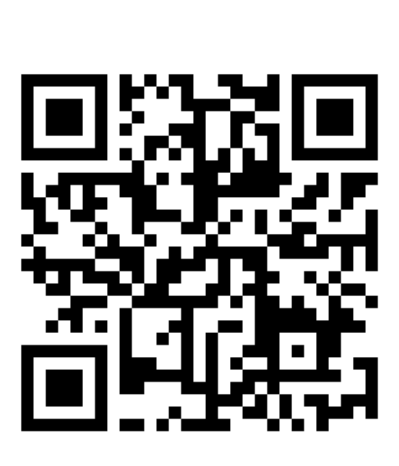

Recibido 02/07/2021

\author{
${ }^{1}$ Dra. Estefanía Ramírez Zumbado \\ Hospital San Vicente de Paul, Heredia, Costa Rica \\ (iD) https://orcid.org/0000-0002-9683-2316
}

${ }^{2}$ Dra. Adriana Acuña Alvarado Hospital Metropolitano, San José, Costa Rica

(i) https://orcid.org/0000-0003-1904-2262

${ }^{3}$ María Fabiola Azofeifa Zumbado Investigadora independiente, Heredia, Costa Rica

(iD https://orcid.org/0000-0002-9376-299X

Corregido $15 / 07 / 2021$
Aceptado
$20 / 07 / 2021$

\section{RESUMEN}

Durante el embarazo el cambio de fisiología y la consideración de riesgos para el feto cambian el manejo del dolor en pacientes con condiciones crónicas. En este artículo se revisan los perfiles de los fármacos utilizados en el manejo del dolor no cancerígeno y como su uso puede cambiar durante el embarazo. Se debe aprovechar a máxima capacidad terapias no farmacológicas para manejar el dolor ya que los perfiles de seguridad de los fármacos son inciertos debido a limitaciones éticas (no se conocen dosis en las que inician efectos teratogénicos, por ejemplo) y el uso de opioides conlleva riesgos importantes (NAS, dependencia, malos resultados fetales o teratogenicidad).

PALABRAS CLAVE: dolor crónico; embarazo; analgésicos; opioides.

\section{ABSTRACT}

During pregnancy, the change in physiology and the consideration of risks to the fetus change pain management in patients with chronic conditions. This article reviews the profiles of the drugs used in the management of non-cancerous pain and how their use may change during pregnancy. Non-pharmacological therapies should be used to full capacity to manage pain since the safety profiles of the drugs are uncertain due to ethical limitations (there are no known doses in which teratogenic effects start, for example) and the use of opioids carries important risks (NAS, dependence, poor fetal outcomes, or teratogenicity).

KEYWORDS: chronic pain; pregnancy; analgesics; opioid. 
${ }^{1}$ Médica general, graduada de la Universidad de Ciencias Médicas (UCIMED). Cód. MED14591. Correo: nia erz@me.com

${ }^{2}$ Médica general, graduada de la Universidad de Ciencias Médicas (UCIMED). Cód. MED14606. Correo: adriac2192@gmail.com

${ }^{3}$ Estudiante de la Ciencias Médicas (UCR). Correo: mafabiola15@gmail.com

\section{INTRODUCCIÓN}

Durante el embarazo el dolor crónico representa un reto clínico. Los cambios farmacocinéticos y la falta de estudios experimentales por problemas éticos y la ausencia de guías clínicas, pueden dificultar la continuación del tratamiento de estas pacientes. Las pacientes corren riesgo de encontrarse submedicadas por temor a perjudicar su embarazo. El fallo terapéutico para manejar el dolor puede llevar a depresión, ansiedad o incluso manifestaciones físicas como hipertensión $(1,2)$, que ponen en riesgo a la madre y al producto.

En este artículo el dolor crónico se define como dolor por más de 6 meses, y se refiere principalmente al diagnosticado previo al embarazo. La revisión tiene como objetivo revisar los perfiles de seguridad de fármacos utilizados en el manejo crónico del dolor y consideraciones importante cuando se reflexiona su uso en personas embarazadas. Es importante recalcar que el manejo del dolor debe ser desde la medicina integrativa y no exclusivamente por tratamiento farmacológico (3). Con este enfoque se puede reducir la dosis mínima de opioides para el manejo del dolor, lo que a su vez reduce las posibilidades de desarrollo de dependencia y síndrome de abstinencia neonatal (SAN) $(1,4)$. El uso de opioides en el embarazo se debe sopesar con cuidado, en estados unidos los opioides se asocian a un $50 \%$ de admisiones por el tratamiento de abuso de drogas durante el embarazo y puede cuadriplicar la mortalidad en el parto (5).

\section{MÉTODO}

Se realizó una revisión bibliográfica sobre el manejo del dolor crónico durante el embarazo a partir de artículos científicos de menos de 5 años de antigüedad publicados en revistas indexadas. Los artículos revisados adicionalmente deben cumplir con los siguientes criterios: buena redacción (claridad), buena organización, buena presentación del material, disponibilidad en inglés o español y las referencias bibliográficas están actualizadas y completas.

Para la recopilación de información se utilizaron las bases de datos PubMed y Scientific Electronic Library Online (SciELO). Las palabras claves utilizadas fueron: dolor crónico, embarazo, analgésicos, opioides.

\section{ANTIINFLAMATORIOS NO ESTEROIDEOS}

Se consideran los fármacos más utilizados en el embarazo, por prescripción médica o automedicados para dolores agudos, resfriados comunes, cefaleas, cuadros gripales (7).

Todos los antiinflamatorios no esteroideos (AINE) son propensos a causar efectos secundarios ya sea en la madre o en el feto, sus diferentes efectos dependen de las isoformas de las ciclooxigenasas que son inhibidas (7). Los inhibidores no selectivos de la COX, se consideran categoría B por la Administración de Alimentos y Medicamentos de los Estados Unidos (FDA). En un estudio de prevención nacional de defectos al nacer, realizado en Boston, en el Departamento de Salud Pública, se evidenció que el $22,6 \%$ de las mujeres con hijos con algún defecto congénito, reportaron consumo de antiinflamatorios no esteroideos durante el embarazo, en su mayoría ibuprofeno, aspirina y naproxeno. (6). 
En la medida de lo posible, se debe de evitar el uso de AINE; sin embargo, si la mujer ha sido expuesta a dichos fármacos, no se considera motivo para interrupción del embarazo. Kallen y Otterblad Olausson demuestran defectos causantes de patologías cardiovasculares leves para casi todos los AINEs estudiados; salvo el naproxeno, donde aumenta significativamente el riesgo de alteraciones cardiacas (7).

Estudios múltiples han relacionado el uso de AINEs con el incremento de anomalías estructurales, entre ellas: hernia diafragmática, defectos tabique interventricular, onfalocele y retraso en el crecimiento intrauterino.

- Acetaminofén (Paracetamol): es el fármaco automedicado más utilizado, para alivio de dolor y como antipirético, se considera seguro durante el embarazo. Un estudio danés que hace referencia a esta droga no encontró relación entre este medicamento y el aborto espontáneo (6), muerte fetal o bajo peso al nacer (8). Se ha relacionado en algunos estudios el uso del Acetaminofén con la gastrosquisis y defectos en saco amniótico (7).

Algunas fuentes de información han vinculado el uso del acetaminofén por parte de la embarazada (8), con el desarrollo de asma en el infante; no obstante, Kallen et al (7) no ha encontrado dicha asociación, aun así, se recomienda evitar el uso excesivo no necesario durante el periodo de embarazo.

En general el Paracetamol se mantiene como la primera línea como analgésico cuando medidas no farmacológicas son insuficientes, la dosis no debe exceder 4 gramos diarios e idealmente no un uso prolongado (8).

- Ácido acetilsalicílico: es el AINE más estudiado, su uso debe de ser evitado en etapas tardías del embarazo, ya que puede aumentar el riesgo de sangrados (por inhibición irreversible de síntesis de prostaglandinas), prolongación de labor de parto, inhibición de la función de las plaquetas neonatales hasta 5 días después del parto y cierre prematuro del ducto arterioso (6). Se prefieren dosis bajas < $150 \mathrm{mg}$ diarios (8).

- Indometacina: se considera un fármaco seguro en tratamiento a corto plazo, no se cuenta con evidencia que respalde el aumento de malformaciones congénitas con su uso durante el primer trimestre de embarazo, por lo que se considera seguro durante este periodo.

Sin embargo, la indometacina debe evitarse después de la semana 32 , debido a que corre riesgo de causar hipotensión pulmonar neonatal significativa, por constricción útero ductal y oligohidramnios (6). otras complicaciones son: ducto arterioso, disfunción renal, enterocolitis necrotizante, perforación intestinal, sangrado intracraneal y lesiones quísticas cerebrales.

A diferencia del ácido acetilsalicílico, la indometacina provoca una inhibición reversible de las prostaglandinas, si se descontinúa una o más semanas antes del parto, no se deberían de esperar complicaciones neonatales ni en labor de parto.

Este fármaco es utilizado para controlar amenazas de parto pretérmino, si este tratamiento falla y el parto ocurre en 48 horas de la toma de indometacina, se aumenta el riesgo de complicaciones neonatales y aumento de morbilidad, por lo que se puede recomendar tocólisis.

- Ibuprofeno: se le han adjudicado defectos congénitos, puede causar disminución del lumen del ducto arterioso (como el naproxeno), lo que puede desarrollar oligohidramnios. No obstante, su uso en el primer trimestre no se ha visto asociado a hipertensión 
pulmonar persistente en el recién nacido (6).

También se ha reportado una reducción en la mineralización de las vértebras lumbares luego de dosis elevadas de ibuprofeno (6).

- Inhibidores selectivos de COX 2: se cuenta con información limitada de esta familia de fármacos y son considerados como categoría $\mathrm{C}$ durante el embarazo (6).

\section{OPIOIDES}

Se considera su uso en el tratamiento de dolor de moderado a severo. (9). El Estudio de Prevención de Defectos al Nacimiento y el estudio de Defectos al Nacimiento del Centro Epidemiológico Slone, señalan asociación entre consumo de opioides por parte de la madre y algunas malformaciones en el recién nacido, el primero, correlaciona defectos como espina bífida, cardiovasculares y gastrosquisis, con énfasis en la codeína e hidrocodona. El segundo estudio, de la misma manera, denota defectos en el tubo neural (7). No obstante, al igual que la mayoría de los medicamentos y su uso en el embarazo, necesita de más estudios para poder establecer los efectos directos en el feto y la madre. Aunque estudios importantes como los mencionados encuentran relaciones entre los opioides y teratogenicidad, en revisiones sistemáticas se puede evidenciar que los estudios son inconclusos (10). Se deben recetar con precaución en mujeres que puedan quedar embarazadas (recetar con método anticonceptivo seguro) y en etapas tempranas del embarazo, y en cada etapa considerar los riesgos y beneficios de la dosis (1).

También se asocia el uso de opioides con SAN (11). El síndrome se caracteriza por dificultades para alimentarse, problemas respiratorios, irritabilidad, hipertonía, insomnio y convulsiones (12). Los opioides cruzan la placenta rápidamente ya que la mayoría son lipofílicos y se unen a receptores mu como agonistas; por esta razón los agentes de liberación prolongada no se recomiendan durante el embarazo. Aumentan la posibilidad de acumulación placentaria y fetal (1).

La Guía Canadiense para el Uso de Opioides en el Tratamiento de Dolor Crónico no Canceroso, ha publicado las siguientes recomendaciones (14):

- Los opioides deben de ser titulados o descontinuados, o bien otorgar la dosis mínima posible.

- En el periodo postparto, el uso de codeína debe de ser evitado o limitado a 4 días.

- Las mujeres con uso indiscutible de opioides deben de llevar control por perinatólogo.

- Mujeres con patologías que ameritan uso de opioides, deben de ser referidas para un tratamiento apropiado.

Dentro de los opioides, el tramadol, es considerado categoría C durante el embarazo y en la analgesia de labor de parto, no afecta la contractilidad uterina ni induce depresión respiratoria en el recién nacido (6).

Con respecto a la codeína, también es considerado categoría C por la FDA, Nezvalov y Henriksen no encontraron aumentos significativos en las malformaciones congénitas; sin embargo, si lograron correlacionarla exposición de codeína y parto prematuro por cesárea, entre niños expuestos y no expuestos, en el tercer trimestre de embarazo, también demostraron el aumento de hemorragia postparto, por lo que se debe de usar con precaución durante el embarazo (6). Adicionalmente, la codeína es el opioide con el que asocia un mayor riesgo de SAN (1).

Con respecto a los opioides sintéticos hay incertidumbre (7), no obstante, el fentanilo, morfina, tramadol e hidromorfona, son drogas efectivas y seguras en situaciones 
necesarias (6). Respecto a la morfina, se debe resaltar que es la más afectada la fármaco cinética durante el embarazo, aumenta la tasa de aclaramiento, disminuye su vida media y tiene un transporte placentario rápido (1).

Es importante recalcar que una vez se ha alcanzado el manejo del dolor, la dosis para mantener el efecto generalmente aumenta conforme avanza el embarazo. El aumento del volumen sanguíneo, la aceleración del metabolismo y aclaramiento acelerado son factores importantes por considerar. Con dosis altas en el tercer trimestre se puede dividir la dosis para contrarrestar la rápida pérdida de circulación del fármaco activo (15).

\section{COADYUVANTES}

\section{- Ansiolíticos y antidepresivos}

El uso de antidepresivos tricíclicos e ISRS puede ayudar a controlar el dolor con una dosis menor de opioides (1). Sin embargo, como efecto de clase, su uso en el tercer trimestre se ha asociado con SAN e hipertensión pulmonar persistente (1).

\section{- Benzodiazepinas}

Están dentro de los fármacos más utilizados en la población general como ansiolíticos, hipnóticos, antiepilépticos 0 relajantes musculares, su uso para el manejo del dolor es junto a opioides. En el embarazo se han asociado con aumento de incidencia de abortos espontáneos y en algunos estudios animales con paladar hendido $(1,6)$. Su uso está contraindicado hasta el cuarto mes de embarazo y solo se admite uso esporádico, ya que su uso prolongado se puede asociar con el síndrome del niño hipotónico, bajo Apgar e hipotermia con síndrome de abstinencia, por lo que al acercarse a la fecha de parto deben de ser descontinuados.

Se debe utilizar con mucha precaución ya que se ha asociado su uso conjunto con opioides se ha asociado con un empeoramiento del NAS y con depresión respiratoria materna $(1,12)$.

\section{Figura 1. Uso escalonado de analgésicos durante el embarazo}

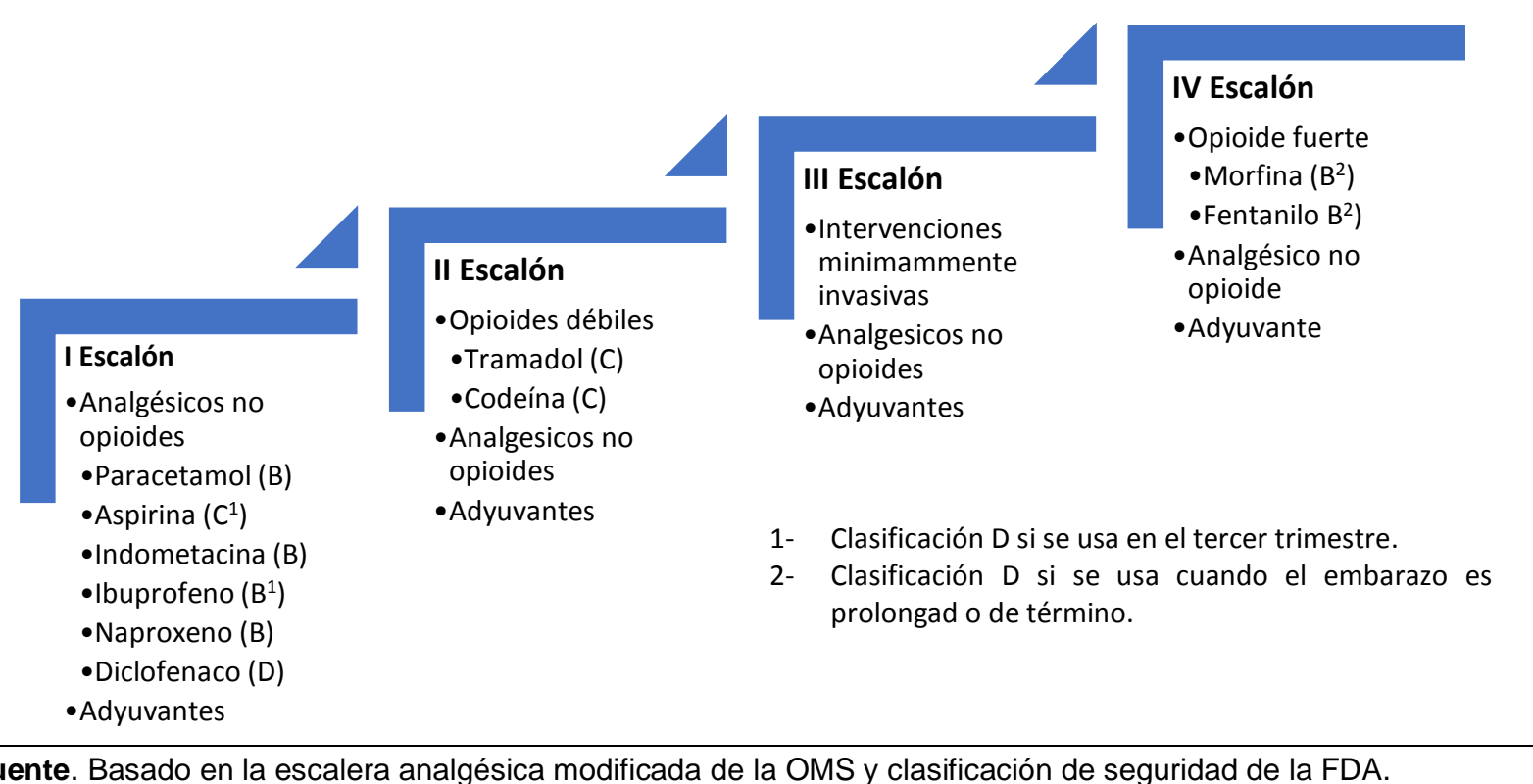




\begin{tabular}{|c|c|c|c|}
\hline Fármaco & $1^{\text {er }}$ trimestre & $2^{\text {do }}$ trimestre & $3^{\text {er }}$ trimestre \\
\hline Acetaminofén & Considerado seguro & Considerado seguro & Considerado seguro \\
\hline $\begin{array}{l}\text { Acido acetil } \\
\text { salicílico }\end{array}$ & $\begin{array}{l}\text { Estudios } \quad \text { lo } \\
\text { relacionan con } \\
\text { riesgo de sangrado y } \\
\text { gastrosquisis, pero } \\
\text { evidencia es limitada }\end{array}$ & $\begin{array}{l}\text { aumenta el riesgo de } \\
\text { sangrado a dosis } \\
\text { analgésicas, se debe } \\
\text { de disminuir la dosis a } \\
\text { partid de este } \\
\text { trimestre para } \\
\text { prevenir morbilidad y } \\
\text { mortalidad por } \\
\text { preeclampsia }\end{array}$ & $\begin{array}{l}\text { aumenta el riego de } \\
\text { sangrados, } \\
\text { prolongación de labor de } \\
\text { parto, prolongación de } \\
\text { labor de parto, inhibición } \\
\text { de la función de las } \\
\text { plaquetas neonatales } \\
\text { hasta } 5 \text { días después del } \\
\text { parto y cierre prematuro } \\
\text { del ducto arterioso. }\end{array}$ \\
\hline Indometacina & $\begin{array}{l}\text { Considerado seguro } \\
\text { a corto plazo. }\end{array}$ & $\begin{array}{l}\text { considerado seguro a } \\
\text { corto plazo }\end{array}$ & $\begin{array}{l}\text { Se debe de evitar a partir } \\
\text { de la semana } 32 \text { por el } \\
\text { riesgo de hipertensión } \\
\text { pulmonar neonatal } \\
\text { significativa, por } \\
\text { constricción ductal y } \\
\text { oligohidramnios. } \\
\text { También relacionado } \\
\text { con cierre prematuro del } \\
\text { ducto arterioso. }\end{array}$ \\
\hline Ibuprofeno & $\begin{array}{l}\text { Incrementa el riesgo } \\
\text { de } \\
\text { espontáneo } \\
80 \%\end{array}$ & Considerado seguro & $\begin{array}{l}\text { Uso después de las } 30 \\
\text { semanas está } \\
\text { contraindicado, por } \\
\text { posible disminución del } \\
\text { lumen de conducto } \\
\text { arterioso. reducción de } \\
\text { mineralización de } \\
\text { vertebra e s lumbares }\end{array}$ \\
\hline $\begin{array}{l}\text { Inhibidores } \\
\text { selectivos de la } \\
\text { COX } 2\end{array}$ & $\begin{array}{l}\text { Información limitada, } \\
\text { categoría C durante } \\
\text { el embarazo }\end{array}$ & $\begin{array}{l}\text { Información limitada, } \\
\text { categoría C durante } \\
\text { el embarazo }\end{array}$ & $\begin{array}{l}\text { Información limitada, } \\
\text { categoría C durante el } \\
\text { embarazo }\end{array}$ \\
\hline Opioides & $\begin{array}{l}\text { usar con precaución, } \\
\text { aumento de SAN }\end{array}$ & $\begin{array}{l}\text { Usar con precaución, } \\
\text { aumento de SAN }\end{array}$ & $\begin{array}{l}\text { se ha correlacionado } \\
\text { con parto prematuro por } \\
\text { cesárea, hemorragia } \\
\text { postparto, aumento de } \\
\text { SAN }\end{array}$ \\
\hline
\end{tabular}




\begin{tabular}{|c|c|c|c|}
\hline Morfina & $\begin{array}{l}\text { Aumento de volumen } \\
\text { sanguíneo, } \\
\text { metabolismo, } \\
\text { aumento de volumen } \\
\text { de aclaramiento, } \\
\text { disminución de la } \\
\text { vida media y rápido } \\
\text { transporte } \\
\text { transplacentario, } \\
\text { aumentan los } \\
\text { requerimientos de } \\
\text { dicho medicamento }\end{array}$ & & \\
\hline Codeína & $\begin{array}{l}\text { Posible aumento de } \\
\text { riesgo de defectos } \\
\text { cardiacos }\end{array}$ & Considerado seguro & Considerado seguro \\
\hline $\begin{array}{l}\text { Fentanilo, } \\
\text { tramadol, } \\
\text { hidromorfina }\end{array}$ & $\begin{array}{l}\text { Efectivas y seguras } \\
\text { en situaciones } \\
\text { necesarias } \\
\text { * tramadol: aumenta } \\
\text { el riesgo de aborto } \\
\text { espontáneo }\end{array}$ & $\begin{array}{l}\text { Efectivas y seguras } \\
\text { en } \\
\text { necesarias }\end{array}$ & $\begin{array}{l}\text { Efectivas y seguras en } \\
\text { situaciones necesarias }\end{array}$ \\
\hline ISRS & $\begin{array}{l}\text { Se desconoce riesgo } \\
\text { de teratogenicidad }\end{array}$ & $\begin{array}{l}\text { Se desconoce riesgo } \\
\text { de teratogenicidad }\end{array}$ & $\begin{array}{l}\text { Su uso se asocia con } \\
\text { SAN e hipertensión } \\
\text { pulmonar persistente }\end{array}$ \\
\hline $\begin{array}{l}\text { Antidepresivos } \\
\text { tricíclicos }\end{array}$ & $\begin{array}{l}\text { Se desconoce riesgo } \\
\text { de teratogenicidad }\end{array}$ & $\begin{array}{l}\text { Se desconoce riesgo } \\
\text { de teratogenicidad }\end{array}$ & $\begin{array}{l}\text { Riesgo de SAN si se } \\
\text { usan en tercer trimestre }\end{array}$ \\
\hline Benzodiacepinas & $\begin{array}{l}\text { Hasta el cuarto mes, } \\
\text { su uso se relaciona } \\
\text { con paladar hendido, } \\
\text { labio leporino y } \\
\text { aumenta la } \\
\text { incidencia en abortos } \\
\text { espontáneos }\end{array}$ & $\begin{array}{l}\text { uso prolongado se } \\
\text { puede asocias con el } \\
\text { síndrome de niño } \\
\text { hipotónico, bajo } \\
\text { APGAR, hipotermia y } \\
\text { SAN }\end{array}$ & $\begin{array}{l}\text { uso prolongado se } \\
\text { puede asocias con el } \\
\text { síndrome de niño } \\
\text { hipotónico, } \\
\text { APGAR, hipotermia y y } \\
\text { SAN }\end{array}$ \\
\hline
\end{tabular}

ABREBIATURA. SAN, síndrome de abstinencia neonatal; ISRS, inhibidores selectivos de la recaptación de serotonina.

FUENTE. Pritham U, Mckay L. Safe management of chronic pain in pregnancy in an era of opioid misuse and abuse

\section{CONCLUSIONES}

El enfoque principal en el manejo del dolor crónico durante el embarazo debe ser un abordaje multidisciplinario y desde medicina integrativa. Se debe aprovechar a máxima capacidad terapias no farmacológicas para manejar el dolor ya que los perfiles de seguridad de los fármacos son inciertos (no se conocen dosis en las que inician efectos teratogénicos, por ejemplo) y conllevan 
riesgos importantes (SAN, dependencia, etc.).

\section{Los autores declaran no tener conflicto de interés.}

\section{REFERENCIAS}

1. Pritham U, McKay L. Safe Management of Chronic Pain in Pregnancy in an Era of Opioid Misuse and Abuse. JOGNN. 2014 May; 43: 554-567.

2. Raymond B, Kook B, Richardson M. The opioid epidemic and pregnancy: implications for anesthetic care. Curr Opin Anaesthesiol. 2018 Jun; 31 (3): 243-250.

3. Yang J, Bauer B, Wahner-Roedler D, Chon T, Xiao L. The Modified WHO Analgesic Ladder: Is It Appropriate for Chronic Non-Cancer Pain?. J Pain Res. 2020 Feb 17; 13: 411-417.

4. Haycraft A. Pregnancy and the Opioid Epidemic. J Psychosoc Nurs Ment Health Serv. 2018 Mar 1; 56 (3): 19-23

5. Martin C, Terplan M, Krans E. Pain, Opioids, and Pregnancy: Historical Context and Medical Management

6. Coluzzi F, Valensise $H$, Sacco $M$, Allegri $M$. Chronic pain management in pregnancy and lactation. Minerva Anestesiol. 2014;80(2):211224.

7. Källén, B. and Reis, M., 2016. Ongoing Pharmacological Management of Chronic Pain in Pregnancy. Drugs, 76(9), pp.915-924.

8. Black E, Khor K, Kennedy D, Chutatape A, Sharma S, Vancaillie T et al. Medication Usa and Pain Management in Pregnancy: A Critical Review. Painn Pract. 2019 Nov; 19 (8): 875-899.

9. Ray-Griffith S, Wendel M, Stowe Z, Magann E. Chronic pain during pregnancy: a review of the literature. International Journal of Women's Health. 2018;Volume 10:153-164.

10. Lind J, Interrante J, Ailes E, Gilboa S, Khan S, Frey $M$ et al. Maternal Use of Opioids During Pregnancy and Congenital Malformations: A Systematic Review. Pediatrics. 2017 Jun; 139 (6): e20164131.

11. Martin C, Terplan M, Krans E. Pain, Opioids, and Pregnancy. Clinics in Perinatology. 2019;46(4):833-847.

12. Patrick S, Cooper W, Davis M. Prescribing opioids and psychotropic drugs in pregnancy. BMJ. 2017 Aug 2; 358

13. Fältmarch S, Perttilä I, Tuomi U, Kautiainen $H$, Gissler M, Pennanen P et al. Use of opioids during pregnancy and effects of pregnancy outcomes. Pharmacepidemiol Drug Saf. 2019 Sep; 28 (9): 1239-1245
14. Tucker Edmonds B, McKenzie F, Austgen M, Ashburn-Nardo L, Matthias M, Hirsh A. Obstetrical Providers' Management of Chronic Pain in Pregnancy: A Vignette Study. Pain Medicine. 2016;:832-841.

15. Blandthorn J, Leung L, Loke Y, Martyn D, Thurman R, Bowman $E$ et al. Prescription opioid use in pregnancy. Aust N Z J Obstet Gynaecol. 2018 Oct; 58(5): 494-498 